\title{
The Role of Uterine Artery Doppler Sonography in Predicting Pre Eclampsia at 14-20 Weeks of Gestation
}

\author{
Dr. (Brig) K. Sahoo, Dr. Pramod Shaha, Dr. Shweta Bhairagond, Dr. Vinay Raj R. \\ Krishna Institute of Medical Sciences University
}

\begin{abstract}
Objective: The purpose of this study was to determine the role of uterine artery Doppler flow velocimetry at second trimester of pregnancy in prediction of preeclampsia. Methods: This prospective study is carried out in the department of Radiodiagnosis, Krishna institute of Medical science, Karad. The study includes 220 pregnant women (primigravida) from August 2013 to September 2014 whose Uterine artery resistive index and pulsatility index was calculated between 14 to 20 weeks of pregnancy. The main uterine artery was located at the uterocervical junction close to the cross- over point of the uterine and external iliac arteries on both sides. RI $>0.7$ \& PI $>1.4$ was considered abnormal. Conclusion: Doppler velocimetry of uterine artery at second trimester of pregnancy can be used as a reliable screening test for prediction of preeclampsia and helps to identify women at high risk and helps to modify follow up and management.
\end{abstract}

Keywords: Resistivity index, Pulsatility index

\section{Introduction}

Even in the era of modern obstetrics, preeclampsia remains as a major complication of pregnancy which can lead to significant incidences of maternal and neonatal mortality and morbidity. Despite advances in medical research, reliable screening test for prediction of this adverse complications still lacking.

Pre-eclampsia is heterogeneous disorder with variable maternal and fetal manifestations. ${ }^{1}$ Pre-eclampsia complicates $3 \%-8 \%$ of pregnancies worldwide ${ }^{2}$. Overall 10 $15 \%$ of maternal deaths are associated with preeclampsia and eclampsia ${ }^{2}$.

In the mother, pre-eclampsia may cause premature cardiovascular disease, such as chronic hypertension, ischemic heart disease and stroke later in life, while children born after preeclamptic pregnancies and who are relatively small at birth, have an increased risk of stroke, coronary heart disease and metabolic syndrome in adult life ${ }^{2}$.

Pre-eclampsia is defined as the development of hypertension ,proteinuria, or both, after 20 week in a women with previously normal blood pressure. It may be associated with many other signs and symptoms such as edema, visual disturbances, headache, and epigastric pain ${ }^{3}$.

The fundamental cause of pre-eclampsia is thought to be the abnormal uteroplacental circulation resulting from the failure of second wave of trophoblastic invasion into spiral arterioles, this will result in increased resistance to flow within the uterine arteries and decreased plancental perfusion as well as due to imbalance between prostacycline \& thrombaxane A2 production..

The crucial issue to understand is that the prime mover of pre-eclampsia is abnormal placentation. Two common theories appear to be interlinked, ie, a genetic theory and an immunological theory.
Pre-eclampsia can be perceived as an impairment of the maternal immune system that prevents it from recognizing the feto-placental unit.

This concept has led to the idea of using Doppler assessment of uterine artery flow velocity waveform as a screening test for predicting preeclampsia ${ }^{4}$. Doppler examination of uterine arteries is a non invasive tool that can be used to indirectly assess trophoblast development and uteroplacental perfusion.

Uterine artery Doppler sonography done during second trimester can accurately predict pre-eclampsia ${ }^{1}$. Resistance Index (RI), pulsatility index which are considered indirect measures of uteroplacental circulation.

Criteria to diagnosis preeclampsia ${ }^{2}$.

Systolic blood pressure $>140 \mathrm{mmHg}$ Two readings of diastolic blood pressure of more than $90 \mathrm{mmHg}$ or higher. Proteinuria of more than $30 \mathrm{mg}$ in a minimum of two random urine samples ${ }^{2}$.

A high resistance index and persistent uterine artery notching, pulsatility index in uterine artery Doppler wave form has shown as best screening test.

So this study is intended to study the validity of uterine artery Doppler sonography to predict pre-eclampsia and to allow judicious allocation of resources for monitoring and preventive treatment to improve maternal and perinatal outcomes 5 .

\section{Pathophysiology of Preeclampsia}

Pre-eclampsia is a pregnancy specific syndrome of reduced organ perfusion secondary to vasospasm and endothelial activation. Minimum criteria for preeclampsia are $^{3}$ : 


\section{International Journal of Science and Research (IJSR) \\ ISSN (Online): 2319-7064 \\ Index Copernicus Value (2013): 6.14 | Impact Factor (2014): 5.611}

1. BP140/90mm Hg after 20 weeks gestation

2. Proteinuria $300 \mathrm{mg} / 24$ hours or $1+$ dipstick

The syndrome complexes of pre-eclampsia and fetal growth restriction have similar pathology of placental insufficiency. Here the blood supply to the fetus is inadequate because of defective placentation, which can be part of syndrome of preeclampsia or can individually lead to defective growth of the baby i.e. small for gestational age baby.

Normal growth and development of uteroplacental circulation during pregnancy: During the first 12 weeks of pregnancy cytotrophoblast invade the spiral arterial walls in the decidua and replace the endothelium and muscular media with a matrix of cytotrophoblasts and fibrinoid and fibrous tissue $^{6,7}$. The fibrinoid material is a complex of maternal fibrin and other plasma constituents plus proteinaceous material derived from the trophoblastic cells. Beginning at about 12 weeks of gestation and continuing throughout the remainder of the second trimester, the endovascular trophoblast move in to the myometrial segments of spiral arteries. Once again the trophoblast replaces the endothelium and establishes themselves in the muscular media. The elastic and muscular tissue of the myometrial segments of the spiral arteries is gradually lost and replaced with fibrinoid material. This condition, along with increase in blood flow and the associated haemodynamic forces, convert the entire length of the spiral arteries from small muscular arteries to dilated, tortuous uteroplacental vessels. At term these changes can be seen at the distal portion of the radial arteries. In all, approximately 100-150 converted spiral arteries supply the placental bed. There is increase in flow from $100 \mathrm{ml} / \mathrm{min}-800 \mathrm{ml} / \mathrm{min}$.

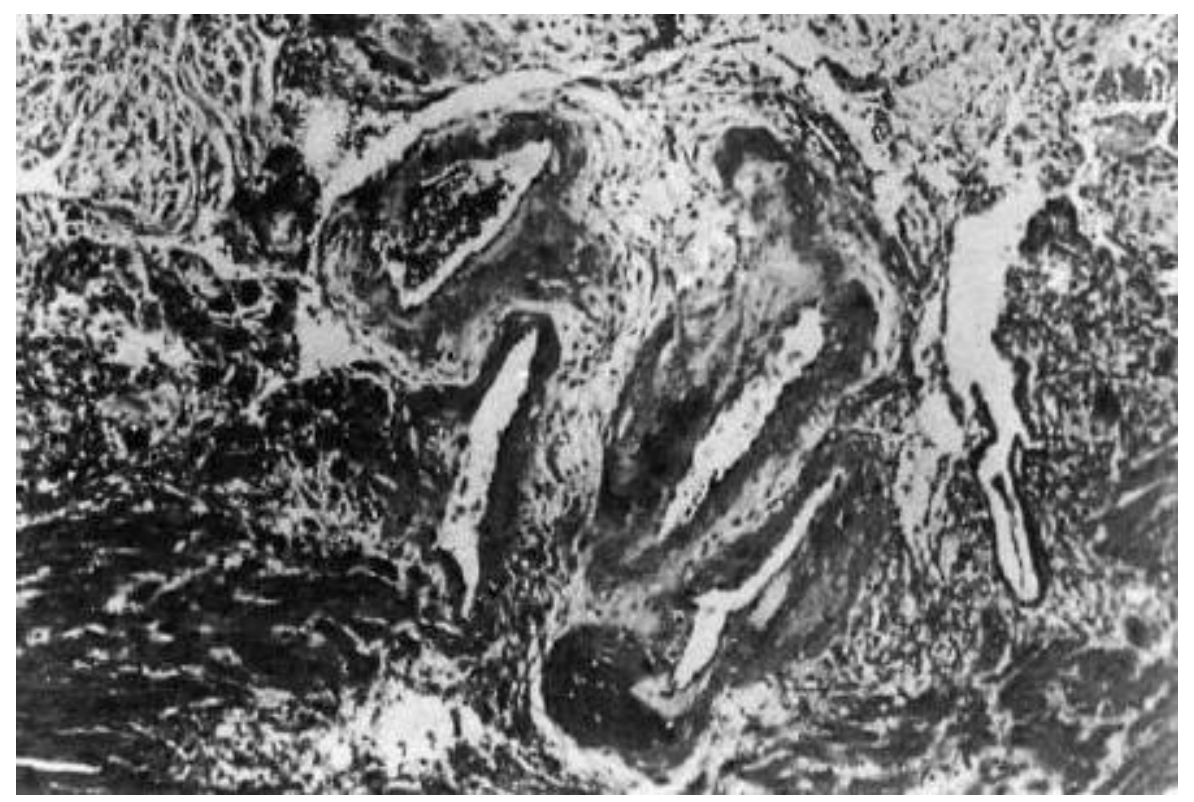

Figure 7: Normal pregnancy. Spiral artery at the myometrial junction shows extensive structural alteration (physiological changes).

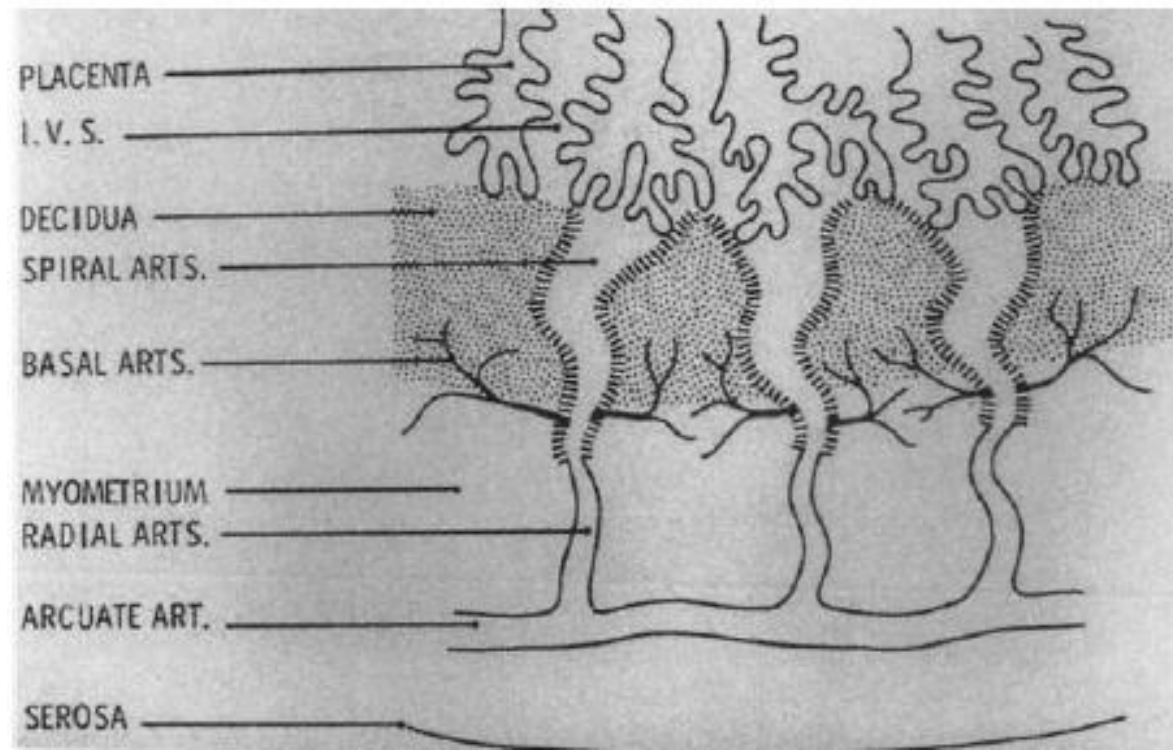

Figure 8: Fully developed physiological changes in the uteroplacental arteries during normal pregnancy. Hatched portion of the wall of these vessels indicate the extent of the physiological changes. (I.V.S - Intervillous space) 


\section{International Journal of Science and Research (IJSR) \\ ISSN (Online): 2319-7064 \\ Index Copernicus Value (2013): 6.14 | Impact Factor (2014): 5.611}

\section{Abnormal development of uteroplacental circulation in the presence of preeclampsia}

According to Brosenet $\mathrm{al}^{6}$, Robertson et $\mathrm{al}^{7}$ and Khong et $\mathrm{al}^{8}$ a lack of endovascular infiltration by trophoblast into the myometrial portion of the placental bed spiral arteries is a consistent finding in the presence of preeclampsia. Classically it is held that second wave of endovascular trophoblastic invasion that proceeds in myometrial segments of the spiral arteries from about 15 weeks, does not occur in patients who will develop fetal growth restriction or pre- eclampsia. Lack of physiological conversion is not only apparent in the myometrial segments of spiral arteries, but also in the decidual parts of some of the vessels, so that a proportion of spiral arteries completely fail to undergo trophoblastic invasion and physiological changes. Since unconverted vessels retain high resistance / low capacitance properties, the effect on maternal blood supply to the placenta may be dramatically low. These may manifest as impaired growth of the baby or high BP with proteinuria i.e. pre-eclampsia with its complications.

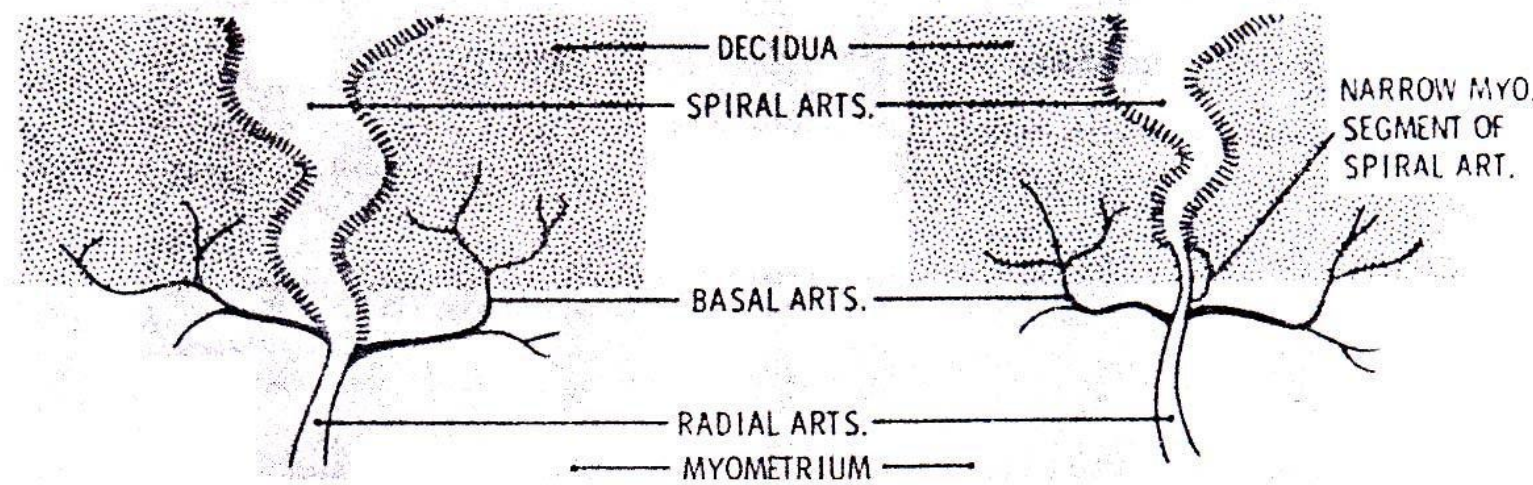

Figure 9: Difference between normal and preeclamptic pregnancies regarding the extent of physiological changes in the uteroplacental arteries

Uteroplacental Circulation - Physiological Changes During Pregnancy.

\section{Non-pregnant uterus}

Characteristics shape of these waveforms shows a steep systolic slope, an early diastolic notch and a small diastolic flow. The waveform remains essentially high resistance although the waveform changes in the menstrual cycle with more flow in the luteal phase. Uterine artery impedance varies according to the phase of ovarian cycle. Kurjaket al.1993 ${ }^{\mathbf{1 0}}$ observed in 150 women that average RI (Resistance Index) during the proliferative phase was $0.88+/-0.04$ (2SD). The RI starts to drop one day prior to ovulation reached a nadir of $0.84+/-0.04$ (2SD) on day 18 and remained at this level for remainder of the cycle. Pregnancy changes were described by Campbell et al, $1983^{11}$. As pregnancy evolves, the diastolic phase augments, the gradient of the deceleration phase reduces, and the notch disappears in the first term in $27 \%$ of pregnancies, although its disappearance is sometimes not simultaneous in both uterine arteries. Flow changes in the uterine arteries are expressed as modification in Doppler indices. At the onset of pregnancy, these indices show few differences compared with their values in the absence of pregnancy. From the 12th-26th weeks of pregnancy there is a progressive lowering of these indices. In addition, the indices are lower in the artery homolateral to the implantation site. The difference between the arteries is more evident from the 8th week and they disappear after the 24th week. The findings are clearly related to the histological changes in the spiral arteries caused by the trophoblastic invasion of these vessels.
During normal pregnancy, the villous cytotrophoblast invades into the inner third of the myometrium, and spiral arteries lose their endothelium and most of their muscle fibers. These structural modifications are associated with functional alterations, such that spiral arteries become lowresistance vessels, and thus less sensitive, or even insensitive, to vasoconstrictive substances ${ }^{2}$.

Pre-eclampsia has a complex pathophysiology, the primary cause being abnormal placentation. Defective invasion of the spiral arteries by cytotrophoblast cells is observed during pre-eclampsia. Recent studies have shown that cytotrophoblast invasion of the uterus is actually a unique differentiation pathway in which the fetal cells adopt certain attributes of the maternal endothelium they normally replace. In pre-eclampsia, this differentiation process goes away ${ }^{2}$.

The abnormalities may be related to the nitric oxide pathway, which contributes substantially to the control of vascular tone. Moreover, inhibition of maternal synthesis of nitric oxide prevents embryo implantation. Increased uterine arterial resistance induces higher sensitivity to vasoconstriction and thus chronic placental ischemia and oxidative stress. This chronic placental ischemia causes fetal complications, including intrauterine growth retardation and intrauterine death.

In parallel, oxidative stress induces release into the maternal circulation of substances such as free radicals, oxidized lipids, cytokines, and serum soluble vascular endothelial growth factor 1 . These abnormalities are responsible for 


\section{International Journal of Science and Research (IJSR) \\ ISSN (Online): 2319-7064 \\ Index Copernicus Value (2013): 6.14 | Impact Factor (2014): 5.611}

endothelial dysfunction with vascular hyper permeability, thrombophilia, and hypertension, so as to compensate for the decreased flow in the uterine arteries due to peripheral vasoconstriction ${ }^{2}$.

Endothelial dysfunction is responsible for the clinical signs observed in the mother, i.e., impairment of the hepatic endothelium contributing to onset of the HELLP (Hemolysis, Elevated Liver enzymes and Low Platelet count) syndrome, impairment of the cerebral endothelium inducing refractory neurological disorders, or even eclampsia ${ }^{2}$. Depletion of vascular endothelial growth factor in the podocytes makes the endotheliosis more able to block the slit diaphragms in the basement membrane, adding to decreased glomerular filtration and causing proteinuria.

Finally, endothelial dysfunction promotes microangiopathic hemolytic anemia, and vascular hyper permeability associated with low serum albumin causes edema, particularly in the lower limbs or lungs ${ }^{2}$.

Pre-eclampsia can be perceived as an impairment of the maternal immune system that prevents it from recognizing the fetoplacental unit. Excessive production of immune cells causes secretion of tumor necrosis factor alpha which induces apoptosis of the extravillous cytotrophoblast.

The human leukocyte antigen (HLA) system also appears to play a role in the defective invasion of the spiral arteries, in that women with pre-eclampsia show reduced levels of HLA-G and HLA-E. During normal pregnancies, the interaction between these cells and the trophoblast is due to secretion of vascular endothelial growth factor and placental growth factor by natural killer cells.

Accordingly, assays of sFlt-1, placental growth factor, endoglin, and vascular endothelial growth factor, all of which increase 4-8 weeks before onset of the disease, may be useful predictors of pre-eclampsia. Recent data show the protective role of hemeoxygenase 1 and its metabolite, carbon monoxide, in pregnancy, and identify this as a potential target in the treatment of pre-eclampsia ${ }^{2}$.

\section{Uterine Artery in PIH Patient}

Impedance to blood flow in the uterine artery may increase in pregnancy complicated by hypertension as shown by Fleschier et al when uterine artery S/D ratio more than 2.6 during third trimester the birth weight at delivery was lower than normal.

Impaired uterine artery flow velocity can be identified by persistent abnormal index, persistent notch and significant difference between the indices in the two vessels. It was demonstrated when the difference between right and left uterine artery S/D ratio is more than one the incidence of adverse fetal outcome is high. Difference right and left artery S/D ratio is probably due to unilateral placentation.

Dehghani-firouzabadi Razieh et $\mathrm{al}^{1}$. in their observational study from October 2011-october 2012 involving 456 pregnant women of 14-16 weeks of gestation where Doppler study was done showed that $\mathrm{RI}(<0.69)$ was observed in 429 patients, who had normal pregnancy outcome. Where as 36 women had RI $>0.69$ had complications of pre eclampsia. They RI can be used to predict pre eclampsia.

In a study conducted by Sanjoy Kumar Bhattacharya et $\mathrm{al}^{4}$. at their center from August 2007 to July 2008 involving 179 patients showed that Doppler velocimetry of uterine artery in second trimester pregnancy can be used as a reliable screening test for prediction of preeclampsia.

Jeltsje S. Cnossen MD, Rachel K. Morris MD et $\mathrm{al}^{2}$. did a systemic review and bivariable metaanalysis of 74 studies of pre-eclampsia found that abnormal uterine artery waveforms are better predictor of pre-eclampsia. Campbell et $\mathrm{al}^{12}$ (1983) was first to report uterine artery Doppler velocimetry they showed that compared to pregnancies with normal uterine artery waveforms, pregnancies with abnormal uterine artery Doppler waveforms were associated with more proteinuric hypertension, required more antihypertensive therapy, and resulted in lower birth weights in younger gestational ages at birth. Thus the capability of this potentially safe noninvasive prospective means of analyzing uterine artery blood flow during pregnancy was realized and set-off a wave of interest and research over the ensuing years.

Kevin Spencer et $\mathrm{al}^{13}$. at their center did a study from October 1999 to August 2002 in 4390 women with singleton pregnancy and observed that uterine artery mean PI improves screening efficacy for prediction of pre-eclampsia. Fabricio da Silva Costa et $\mathrm{al}^{14}$. concluded from his study that second trimester (24-26) is best period to do Doppler uterine artery study.

C. K. H. Yoet $\mathrm{al}^{15}$. conducted the multicentric study involving 30639 pregnancies observed that, pre-eclampsia requiring early delivery is more likely to be associated with small for gestation age than less severe preeclampsia in women who deliver at term. Hence Doppler ultrasound assessment of the uterine arteries is more effective in identifying preeclampsia requiring pre term than term delivery.

\section{The time of examining the uterine flow has been studied} extensively.

Katie $\mathrm{M}$ Groom et $\mathrm{al}^{16}$ described the changes in mean uterine artery resistance index and bilateral uterine artery notches between 20 and 24 weeks of gestation and its outcome was done, concluded that 20 weeks is the most appropriate gestation in second trimester to perform uterine artery doppler.

A.M. Martin et $\mathrm{al}^{17}$ assessed the value of uterine artery doppler at 11-14 weeks of gestational age in the identification of women at risk of developing preeclampsia and fetal growth restriction, concluded that high proportion of women can be screened effectively for pre-eclampsia at 11-14 weeks by doppler study of uterine artery.

K.Harrington et $\mathrm{al}^{18}$ studied trans-vaginal uterine and umbilical artery Doppler at 10 - 12 weeks of gestation, measuring early diastolic notch, resistance index, pulsatility index and subsequent development of pre- eclampsia and IUGR, concluded that abnormal doppler value leads to 


\section{International Journal of Science and Research (IJSR) \\ ISSN (Online): 2319-7064 \\ Index Copernicus Value (2013): 6.14 | Impact Factor (2014): 5.611}

Pregnancy Induced Hypertension (PIH), premature delivery and Small for Gestational Age (SGA) baby.

\section{AIM}

To predict usefulness of uterine artery Doppler in predicting pre-eclampsia and to study uterine artery doppler waveforms.

\section{OBJECTIVE}

1. To determine the role of uterine artery Doppler at 14-20 weeks of gestation.

2. To determine the performance of screening for preeclampsia by maternal uterine artery doppler.

\section{STUDY DESIGN:}

A Prospective study, conducted between August 2013 to August 2015, in the Department of Radiodiagnosis, Krishna hospital, Krishna institute of Medical sciences, Karad.

\section{SAMPLE SIZE AND SOURCE OF DATA}

After assessment of exclusion \& inclusion criteria total 220 women were included into the study the pregnant women of gestational age between $14-20$ weeks attending the OPD at our institution in second trimester pregnancy are included in the study.

\section{INCLUSION CRITERIA}

1. Singleton pregnancy

2. Second trimester pregnancy

3. Primigravida

\section{EXCLUSION CRITERIA}

1. Multiple pregnancy

2. Congenital fetal anamolies

3. Mother who are already hypertensive.

4. If the patient could not be followed till delivery for any reason .

\section{Methods}

The patient was explained in detail about the non invasive nature of the procedure. Synthetic ultra gel was applied over the abdomen to get a good acoustic coupling.
ACUSON X300 SIEMENS Ultrasound machine was used.

Transabdominal uterine artery Doppler will be done using curvilinear probe $(3.5 \mathrm{MHz}$ ) by localizing the uterine artery at uterocervical junction where it appears to cross the external iliac artery. The variable analysed will be .

1. Resistance index(RI) was calculated using formula$\mathrm{RI}=$ Peak systolic velocity - Peak diastolic velocity/ Peak systolic velocity.

2. Pulsatility index (PI) will be calculated using formula.

$\mathrm{PI}=$ Peak systolic velocity - End diastolic velocity / Mean velocity

Subsequent development of preeclampsia can be predicted when resistive index is more than 0.7 and pulsality index is more than 1.4 .

\section{Results and Observation}

Among 220 primigravida patients of singleton pregnancies, with no other pregnancy related problems who underwent Doppler velocitometry, 54 patients had preeclampsia (24.54\%). In preeclampsia patient group RI was greater than $0.7(>0.7$ at

95 percentile) in 42 patients, where as 12 patients had normal RI. Where as PI was $>1.4$ in 24 women only.

Sensitivity, Specificity, Positive predictive value, Negative predictive value were calculated using 2 X 2 table

Table 1: Relationship Between Ri And Preeclampsia

\begin{tabular}{|c|c|c|c|}
\hline $\begin{array}{ll}\mathrm{RI}>0.7 & \mathrm{PE} \\
\end{array}$ & YES & $\mathrm{NO}$ & TOTAL \\
\hline YES & 42 & 24 & 66 \\
\hline $\mathrm{NO}$ & 12 & 142 & 154 \\
\hline TOTAL & 54 & 166 & 220 \\
\hline
\end{tabular}

PE- Preeclampsia, RI- Resistive index

Sensitivity: $77.8 \%$

Specificity: $85.5 \%$

Positive Predictive Value: $63.6 \%$

Negative Predictive Value: 92.2\%

MID $P$ exact value $=<0.0000001$, hence the study is statistically significant.

\section{PREECLAMPSIA ( $\mathrm{N}=54)$ AND RI INDEX}

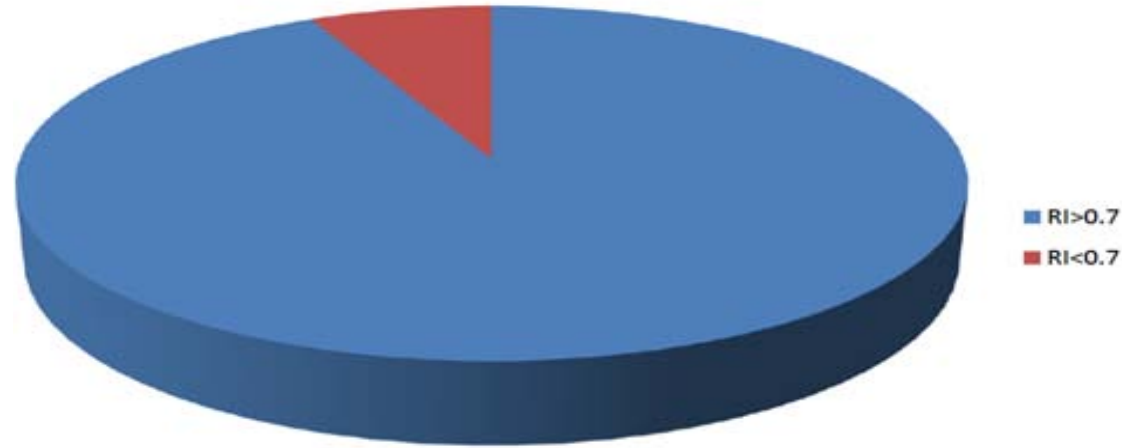

Graph 1: Showing relationship between preeclampsia and high resistive index 


\section{International Journal of Science and Research (IJSR)}

ISSN (Online): 2319-7064

Index Copernicus Value (2013): 6.14 | Impact Factor (2014): 5.611

Table 2: Relationship between PI and Preeclampsia

\begin{tabular}{|l|l|l|l|}
\hline PI $>1.4$ & & & \multicolumn{1}{|c|}{ To } \\
\hline YES & & & \\
\hline NO & 24 & 17 & 41 \\
\hline TOTAL & 30 & 149 & 179 \\
\hline
\end{tabular}

Sensitivity: $44.44 \%$

Specificity: $89.75 \%$

Positive Predictive Value: $58.53 \%$

Negative Predictive Value: 83.24\%

MID P exact value $=<0.0000001$, hence the study id statistically significant.

PE- Preeclampsia, PI- Pulsatility index

\section{PREECLAMPSIA(N=54) AND PI INDEX}

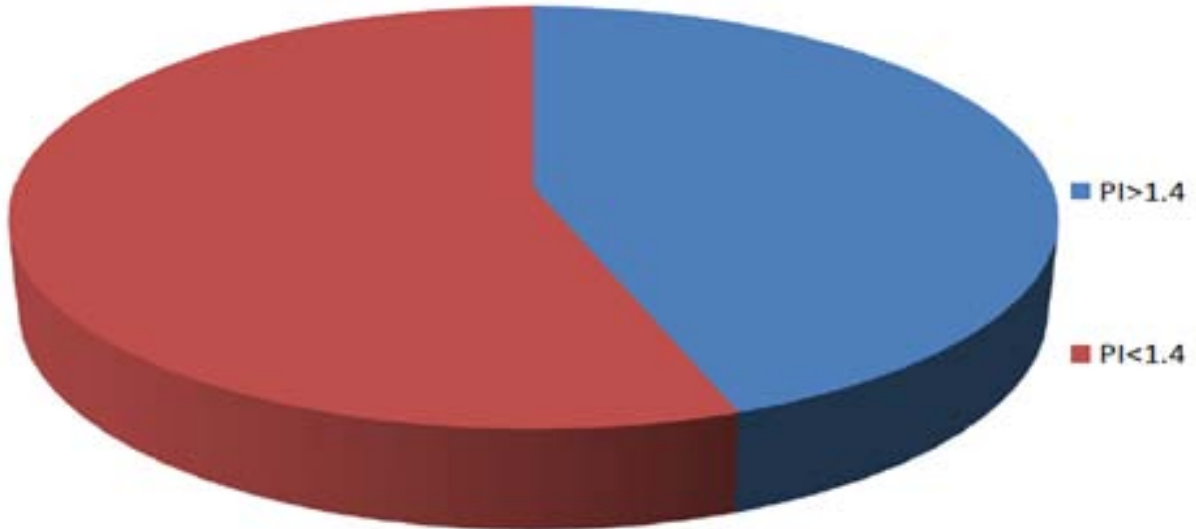

Graph 2: Showing relationship between preeclampsia and high pulsatality index

Table 4 : BP in Study Population

\begin{tabular}{|c|c|c|c|}
\hline BP & Yes & No & Total \\
\hline$>\mathbf{1 4 0 / 9 0 m m ~ o f ~} \mathbf{H g}$ & 98 & 122 & 220 \\
\hline
\end{tabular}

Table 5: Proteinuria In Study Population

\begin{tabular}{|c|c|c|c|}
\hline Proteinuria & Yes & No & Total \\
\hline$>30$ & 110 & 110 & 220 \\
\hline
\end{tabular}

\section{Discussion}

There are few data regarding the uteroplacental circulation doppler pattern during the first and early second trimester of pregnancy. we choose the 14-20 week period to perform the uterine Doppler evaluation because the routine scan for nuchal translucency and early second trimester anatomical assessment was already scheduled at this time of gestation in our population and placental implantation is completed by 14-18 weeks.

Our study found significant hemodynamic changes in uterine artery Doppler assessment during the 14 to 20 weeks of gestation these hemodynamic changes are evident during second trimester likely reflecting a time correlation between the intervillous circulation and marked changes in the umbilical and fetal circulations at this time of pregnancy.

On the basis of our findings and those of others, we speculate that the transabdominal uterine examination in early stages of pregnancy may have technical problems in early gestation. At this stage uterus is not large enough and the full bladder technique is necessary to perform the Doppler examination .consequently the distended bladder may cause an alteration in blood flow in small arteries. By using the transvaginal route, the probe is located closer to the uterine artery, the angle of insonation is usually closer to zero degree and the wave forms obtained have better definition. The pulse Doppler gait can be positioned in the main uterine artery before it branches into the arcuates or other smaller arteries and this can be ascertained more confidently by transvaginal examination.

Our results demonstrated lower impedance in the uterine artery of normal outcome pregnancies than in the complicated cases, suggesting that lack of normal uteroplacental circulation at this early stage of pregnancy may predict the later development of some pregnancy complications.

In past decades, the prediction of pre-eclampsia has been attempted by using several maternal assessment methods; in this respect, Doppler flow studies of the uteroplacental circulation have been extensively performed, since they are easy, inexpensive, non-invasive and can be done in early pregnancy. However, Doppler ultrasound examination of the uteroplacental circulation has provided conflicting results when employed as a screening test for pre-eclampsia or any other hypertensive disorder of pregnancy.

Campbell ${ }^{12}$ et al were pioneers in the presentation of data on uteroplacental circulation by means of the study of arcuate arteries with Doppler velocimetry, having reported that the diastolic notch of uterine arteries at the second and third trimesters of gestation, was related to the increase in uteroplacental vascular resistance, IUGR and hypertension. Current studies report association between RI and PI values. We conducted this prospective study at our institute mainly consisting of rural population. The uterine artery Doppler veocitometry was done in second trimester, the mean RI was calculated and used for analysis. 


\section{International Journal of Science and Research (IJSR) \\ ISSN (Online): 2319-7064 \\ Index Copernicus Value (2013): 6.14 | Impact Factor (2014): 5.611}

In our study when RI index was used, Sensitivity, Specificity, Positive predictive value, Negative predictive value thus obtained were $77.8 \%, 85.5 \%, 63.6 \%, 92.2 \%$ respectively and p value was calculated and it is significant which shows that

RI index can be reliably used for screening for prediction of preeclampsia. When PI index was used Sensitivity, Specificity, Positive predictive value, Negative predictive value were $44.44 \%$, 89.75\%, 58.53\%, 83.24\% respectively and $\mathrm{p}$ value was calculated and it is significant which shows that PI index can be reliably used for screening for prediction of preeclampsia.

Although not in all parameters, the results of some of studies done in the past were comparable to our study and had similar opinion.

Our study results are comparable to study done by Parreetti ${ }^{19}$ et al in their study the use of an individual longitudinal flow pattern indicator resulted in improving accuracy in the prediction of pre-eclampsia as compared with the traditional cross-sectional mean RI at 24 weeks. A further increase in specificity and PPV was obtained using a logit model that includes the longitudinal flow pattern indicator and the cross-sectional RI at 24 weeks. Since both the longitudinal flow pattern indicator and the logit model showed a high performance in predicting pre eclampsia in women with risk factors for impaired placentation, they might be used to identify a high risk population in which preventive measures and/or the therapeutic options might be tested. Longitudinal study using RI as a sole indicator the Sensitivity, Specificity, Positive predictive value, Negative predictive value were $88.9 \%, 82.4 \%$, $62.7 \%$, and $95.4 \%$ respectively.

Our study results are similar to the study done by Bhattacharyya4et $\mathrm{al}^{4} 179$ women were selected, who completed the study. These women were divided into high risk and Low risk groups according to the risk factors, 52 (29.05\%) and 127 (70.95 \%) were in high risk and low risk groups respectively. In low risk group which is similar to our study group, in this group, 10 women developed preeclampsia. Uterine artery velocimetry showed increased RI $(<0.6)$ in 13 women (10.24 \%), and among

them, seven developed preeclampsia. Notching was not seen in any of them. Therefore, the Sensitivity, Specificity, PPV, and NPV of abnormal uterine artery velocimetry become 70 , 94.87, 53.84, and $97.37 \%$, respectively.

Dehghani-firouzabadi Raziehet $\mathrm{al}^{1}$ did doppler studies in 456 pregnant women. A total number of 27 patients showed abnormal uterine artery Doppler finding to develop preeclampsia of the. 23 pregnancies $(85.2 \%)$ of them with preeclampsia had only RI of more than 0.7 and only 4 of them (14.8\%) had $\mathrm{RI}<0.7$ ( $\mathrm{P}=0.001) .26$ (96.3\%) of patients with RI $>0.69$ developed preeclampsia and only 1 patients (3.7\%) with $\mathrm{RI}<0.69$ developed preeclampsia.

Our study results are almost similar to study done by Maryam ${ }^{20}$ et al in predicting Sensitivity, Specificity, Positive predictive value, Negative predictive value for both resistive index and pulsality index.
Results of above said studies show that mid-trimester or second trimester uterine artery Doppler indices like RI\& PI can be useful tool in predicting preeclampsia. And when PI index was used alone the sensitivity was less than RI alone. So RI in second trimester is more sensitive in predicting preeclampsia than PI alone combined with RI and notching.

Specificity and Negative predictive value of our study are similar to study done by Melchioree ${ }^{21}$ et al Sensitivity, Specificity, Positive predictive value, Negative predictive value were 24.2, 95.8, 6, 99.1 respectively, the difference in Sensitivity and Positive predictive value may be due to variable sample sizes.

Our study is similar to Specificity for both RI and PI inJeltsje4et al, since it is multivariate study involving several other studies for analysis, the other parameters are not comparable because, many variables were included in reporting and analysis.

Our study results are similar to study by $\operatorname{Nagar}^{22}$ et al in Specificity, but sensitivity and PPV were low owing to sample size.

F. Crispi et $\mathrm{al}^{23}$ evaluated significance of angiogenic factors and uterine artery doppler studies using PI. They concluded Angiogenic factors and uterine artery Doppler evaluation combined (than individually) may be useful second-trimester screening tests for prediction of preeclampsia.

Accurate early identification of women at risk of preeclampsia could not only dramatically reduce the costs of antenatal care by enabling the identification and close surveillance of women with at-risk pregnancies, but also lead to improved management of hypertensive disorders in later life. There is no widely accepted or accurate method for the early prediction of pre-eclampsia. The present data demonstrate a strong relationship between first-trimester uterine artery Doppler indices and the subsequent development of preterm pre-eclampsia. Even though uterine Doppler imaging may not fulfill the prerequisites for a valid screening test, it may be invaluable in triaging women for increased surveillance.

Although several studies are done for early detection of pre eclampsia using uterine artery doppler, where PI and/or diastolic notching were assessed and had variable results.

But from studies by E. Parretti ${ }^{19}$ et al, K., Melchiorre ${ }^{21}$ et al., Dehghani firouzabadiRazieh1 et al. Bhattacharyya Sanjoy Kumar $^{4}$ et al used RI as main index in their study to determine the adverse outcomes of pregnancy.

Whereas according to Maryam Afrakhteh ${ }^{20}$ etal.the sensitivity for predicting adverse outcomes for mean RI $>95$ th percentile in third trimester was 58\%. It increased to $77.2 \%$ at second trimester. The sensitivity of RI to predict complicated pregnancy in both trimesters was higher than sensitivity of PI and combined RI, PI and bilateral notch. On the other hand, the value of specificity to detect adverse outcome was higher when RI, PI and bilateral notch values were combined. Which suggests the valuable role of second 


\section{International Journal of Science and Research (IJSR) \\ ISSN (Online): 2319-7064 \\ Index Copernicus Value (2013): 6.14 | Impact Factor (2014): 5.611}

trimester ${ }^{\text {ee }}$ s uterine Doppler sonography in screening complicated pregnancy.

In clinical practice, because no single marker effectively predicts the risk of pre-eclampsia, the current trend is to test a combination of markers. The most commonly used combination of markers assesses sFlt-1, placental growth factor, endoglin, and vascular endothelial growth factor during the first or second trimester. Improved prediction of pre-eclampsia has been noticed when serum markers are combined with Doppler indices. Some studies have shown, second trimester maternal serum cystatin C, C-reactive protein, and uterine artery mean resistance index were observed to be independent predictors of pre-eclampsia.

Although delivery is the only effective treatment for preeclampsia. Some of the preventive measures can be taken like early identification, close follow ups and secondary prevention.

One of the secondary prevention is based on antiplatelet aspirin therapy, which reduces the risk of pre-eclampsia by $10 \%$. No study currently allows determination of the exact dosage or the best time for initiation of aspirin. However, aspirin should be

initiated as early as possible.

Our study showed that by detecting abnormal uterine artery Doppler indices(High RI and PI) between 14 to 20 weeks of pregnancy , we can identify women at risk for development of preeclampsia.

This screening study demonstrates the feasibility of Doppler assessment of uterine arteries into the routine scan. our study examined the value of one stage screening at 14 to 20 weeks using Doppler sonography of uterine arteries.

So results of this study can be used to draw the conclusions.

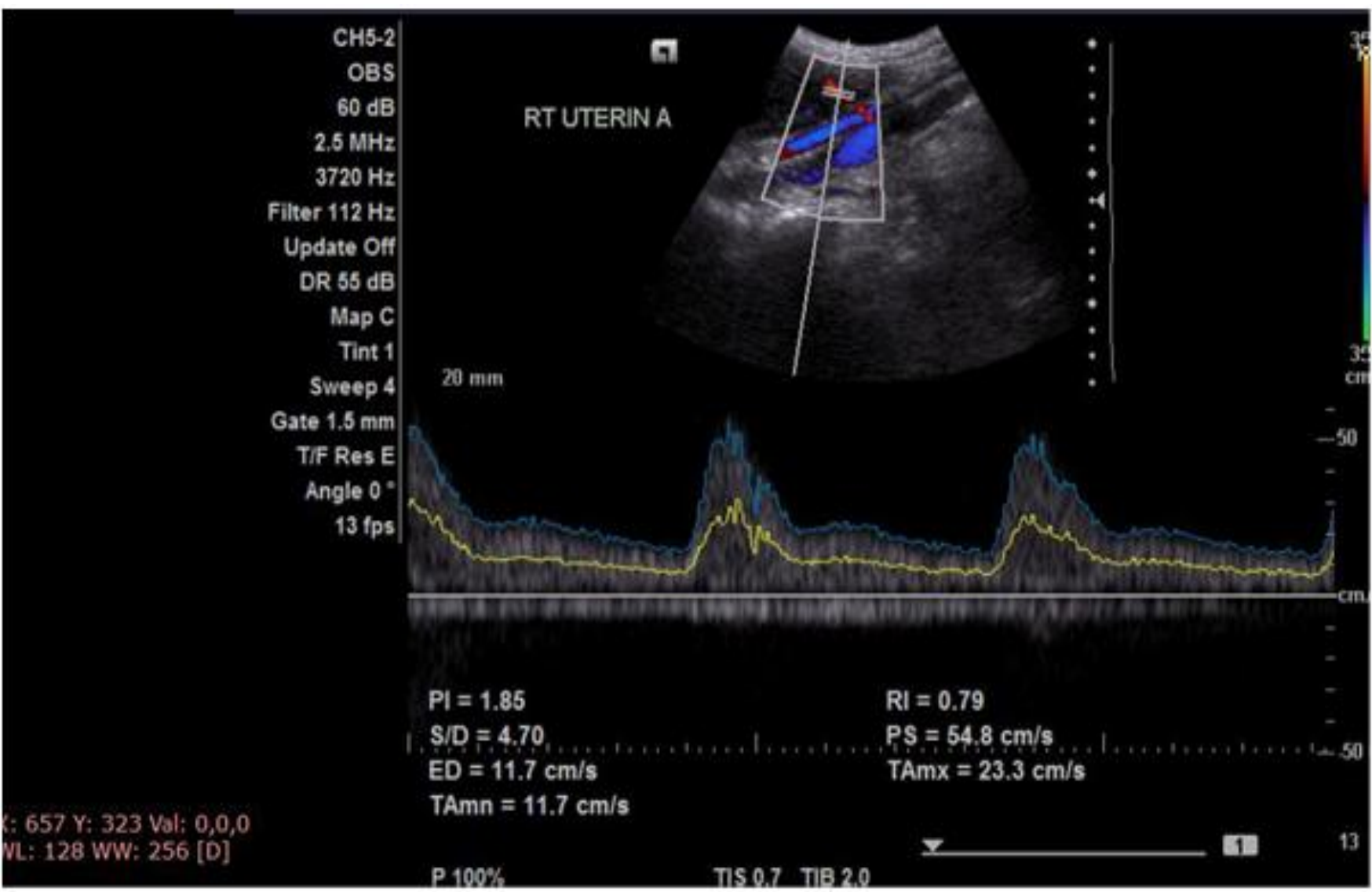

Figure 12: Doppler imaging of right uterine artery showing RI of 0.79 
International Journal of Science and Research (IJSR)

ISSN (Online): 2319-7064

Index Copernicus Value (2013): 6.14 | Impact Factor (2014): 5.611

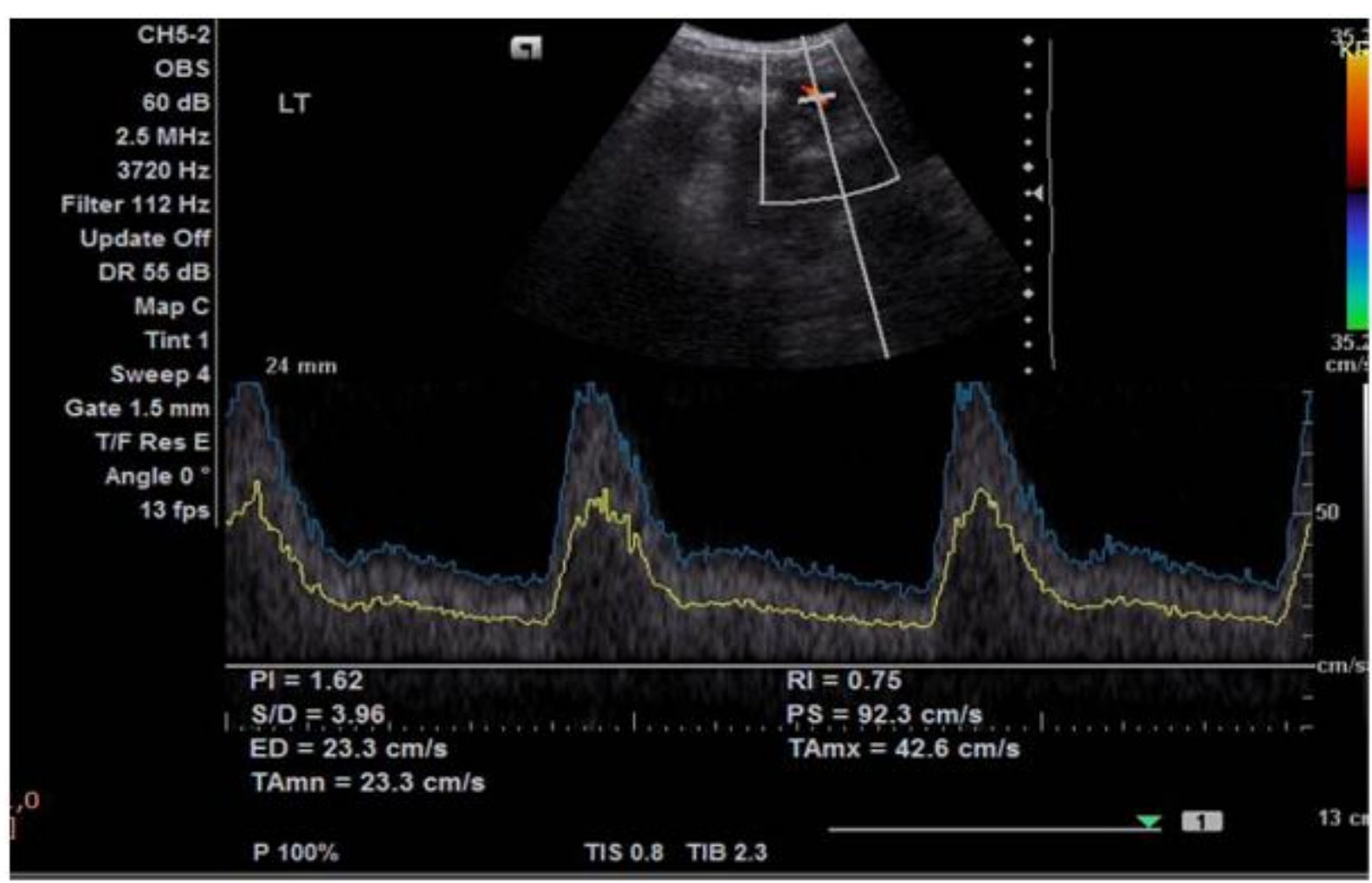

Figure 13: Doppler imaging of left uterine artery showing RI of 0.75

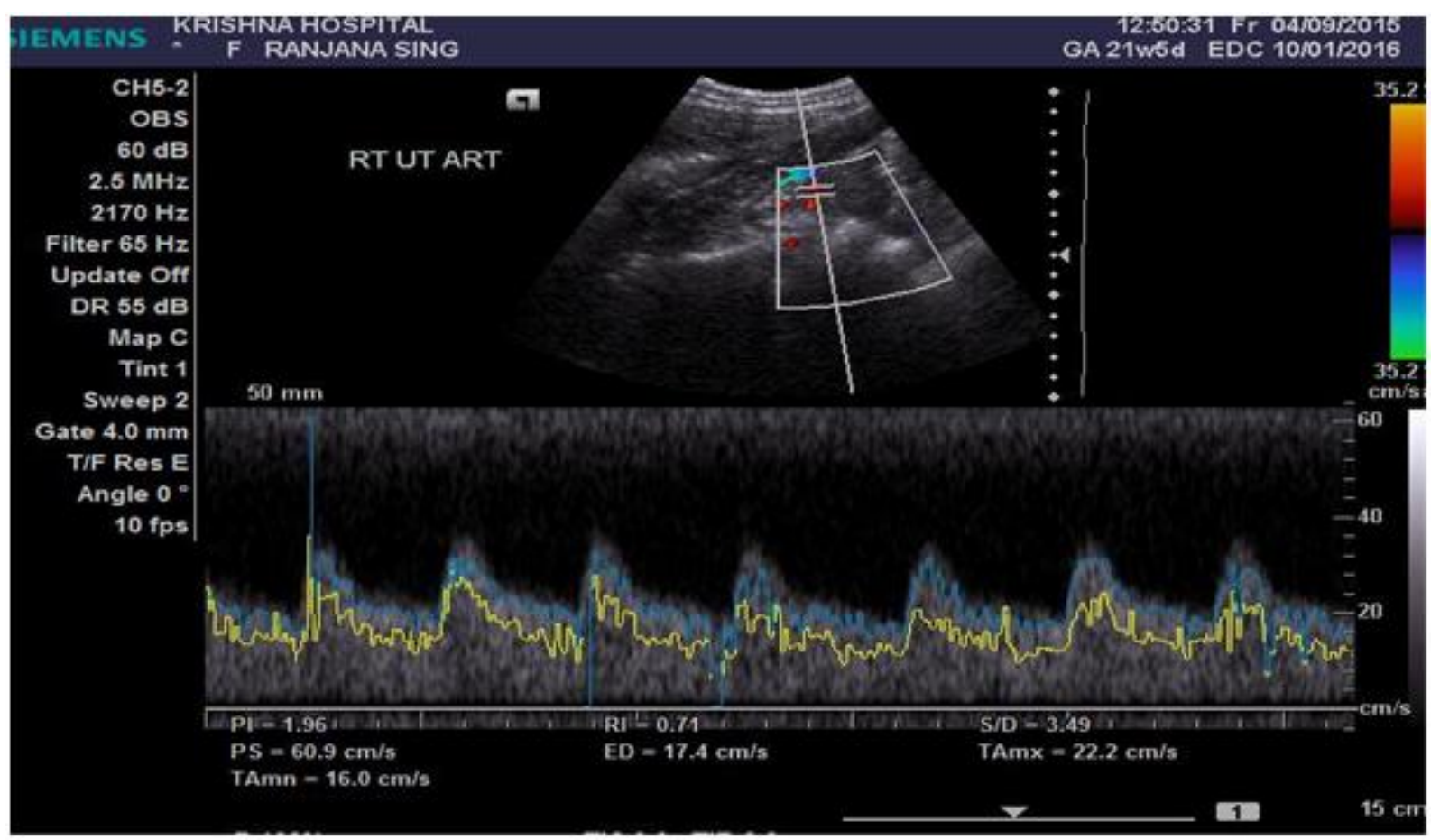

Figure 18: Doppler imaging of right uterine artery showing PI of 1.96

Volume 5 Issue 2, February 2016

www.ijsr.net 


\section{International Journal of Science and Research (IJSR) \\ ISSN (Online): 2319-7064}

Index Copernicus Value (2013): 6.14 | Impact Factor (2014): 5.611

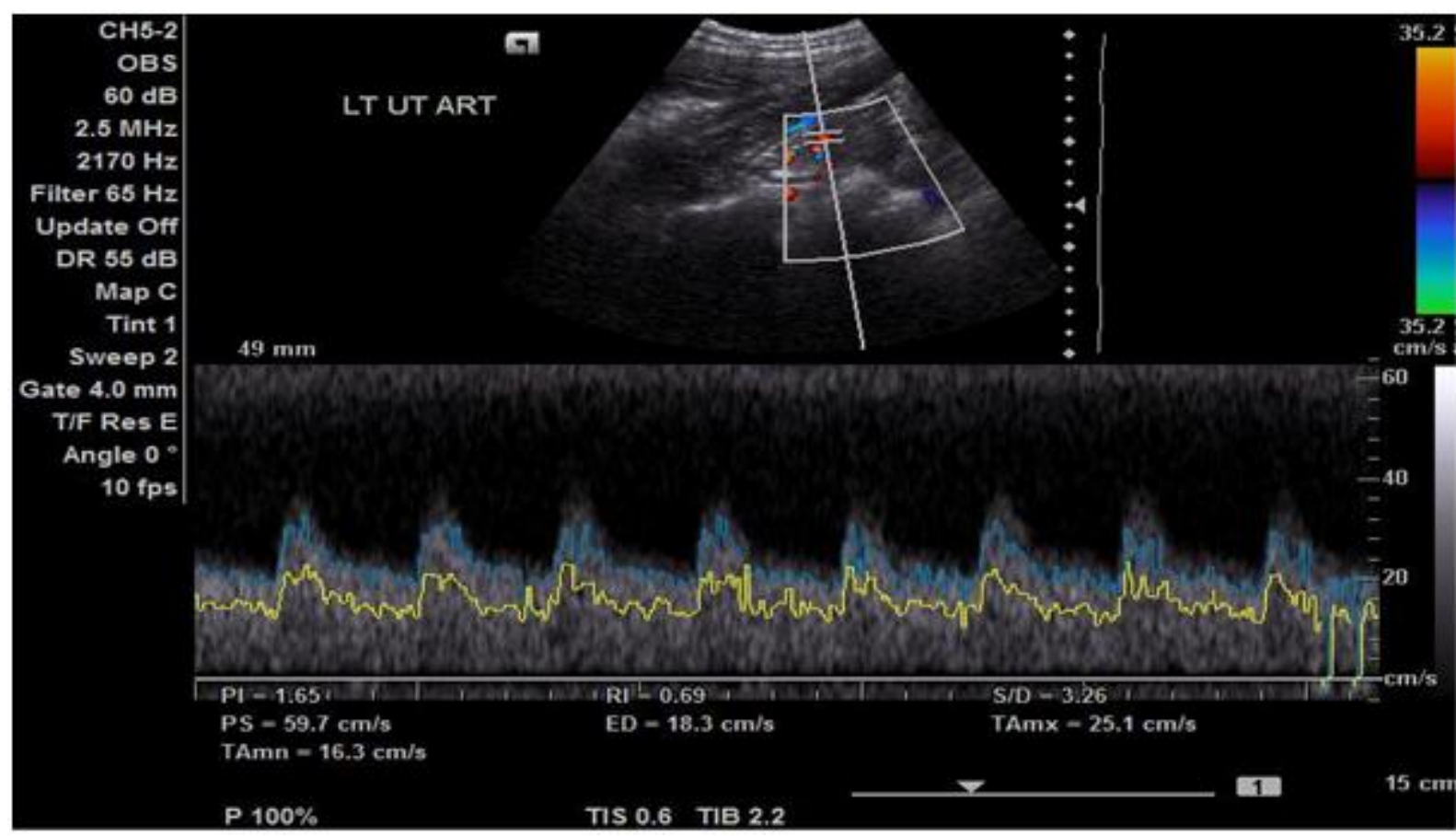

Figure 19: Doppler imaging of left uterine artery showing PI of 1.65

\section{Conclusion}

The introduction of Doppler technology has proved the first opportunity for repetitive, non invasive hemodynamic monitoring in human pregnancy compared to other methods of maternal and fetal monitoring.

Doppler has proved to be more sensitive in detecting early changes in uterine circulation and fetal circulation and aids in the management. It is a primary tool for fetomaternal surveillance in hypertensive pregnancies as the changes in uteroplacental and fetal circulation strongly correlate with pregnancy outcome. Umbilical artery studies should be an integral parameter while evaluating the fetus.

Examination of multiple vessels may add value to decision making. The use of color Doppler is suitable for clinical obstetric practice. Abnormal flow velocity waveforms should encourage closer fetal surveillance with other well established fetal testing modalities.

Timing of delivery with abnormal Doppler depends on gestational age, level of neonatal intensive care, and good clinical judgement with individualization is needed. Both maternal and fetal risk assessment must be taken into consideration.

From results of this study it can be concluded that uterine artery Doppler between 14- 20 weeks of gestation can be used as a reliable screening test for prediction of preeclampsia in cases where the test proves to be abnormal, increased surveillance and delivery in a well equipped setup is necessary to reduce the maternal and fetal complications.

\section{References}

[1] Dehghani-firouzabadi Razieh, Mojibian Mahdyeh et al. 2013. Uterine Artery Doppler Sonography In Predicting
Preeclampsia and IUGR at 14-16 Week Gestation. World Applied Sciences Journal 22 (2):197-201,2013

[2] Jennifer Uzan, Marie Manag. 2011; 7: 467474.Corbonnel et al.2011. Preeclampsia: pathophysiology, diagnosis, management. Vasc Health Risk

[3] Arias practical guide to high risk pregnancy and delivery. A South Asian perspectiev- 4th Edition.

[4] Sanjoy Kumar Bhattacharyya et al.2012. Prediction of Preeclampsia by Midtrimester Uterine Artery Doppler Velocimetry in High Risk and Low Risk Women. J Obstet Gynaecol India. 2012 June; 62(3): 297-300.

[5] Jeltsje S. Cnossen, MD et al. 2008.Use of uterine artery Doppler ultrasonography to predict pre-eclampsia and intrauterine growth restriction: a systemic review and bivariable meta-analysis. CMAJ. 2008 march 11; 178(6): 701-711.

[6] Brosens IA, Robertson WB, Dixon HG (1972). The role of spiral arteries in the pathogenesis of preeclampsia. Obstet Gynecol Annu 1972;1:117-191.

[7] 7.Robertson WB, Brosens I, Dixon G (1975). Uteropcental vascular pathology. Eur J Obstet Gynecol Reprot Biol 1975;5: 47-65.

[8] Khong TY, De Wol F, Robertson WB, et al Inadequate maternal vascular response to placentation in pregnancies complicated by preeclampsia and by smallfor gestational age infants. Br J ObstetGynecol1986; 93:1049- 1059.

[9] Kevin Harrington and Stuart Campbell. The uterine circulation in pregnancy, chapter 4 A colour atlas of Doppler ultrasonography in obstetrics.

[10] Kujrak. A, Seeding, D,funduk-kurjak, B;Shalan, H; Predanic, M; Socic, A Transvaginal color Doppler in the assessment of the uteroplacental circulation in normal early pregnancy. - J Perinat Med. 1993; 21:2534.

[11] Steet CV, Campbell S, Pampigline J, Kingland C, Mason BA, Collins Wp. (1990).Transvaginal color flow 
imaging of the uterine arteries during the ovarian and menstrual cycles. Hum Reprod 1990; 5:391-99.

[12] Campbell S, Diaz Recasens J Griffin DR, et al(1983). New Doppler technique for assessing uteroplacental blood flow. Lancet 1983; 1:675.

[13] Kevin Spencer et al.2005. Prediction of pregnancy compications by firsttrimester maternal serum PAPP-A and free $\beta$-hCG and second-trimester uterine artery Doppler. Prenat Diagn 2005; 25: 949-953.

[14] Fabricio da Silva Costa et al. 2006. Which is best period to perform uterine artery Doppler in prediction of pregnancy complications? Radiol Bras vol.39 no.2 Sao Paulo Mar. / Apr. 2006.

[15]C.K.H. Yoet al.2008. Prediction of preeclampsia by uterine artery Doppler imaging: relationship to gestational age at delivery and small-forgestational age. Ultrasound Obstet Gynecol 2008; 31: 310-313.

[16] Katie MG, Robyn A, North, Peter RS,Eliza H.Y.Chan, Rennae S. Taylor, Gustaaf A.Dekker.et,al. Patterns Of change in uterine artery doppler studies between 20 and 24 weeks of gestation and pregnancy outcome. The American college of obsteticians and Gynoecologist published 2009; 113(2)332-338.

[17]A.M. Martin,R.Bindra,P.Curcio, S.Cicero and K.H.Nicolaides. Screening for preeclampsia and fetal growth restriction by uterine artery doppler at 11-14 weeks of gestation. Ultrasound obstet gynecol 2001;18:583-586.

[18] K.Harrington,C.Goldfrad,R.G.Carpenter and S.Campbell. Transvaginal uterine and umblical artery doppler examination of 12 to 16 weeks and the subsequent developmnet of pre -eclampsia and intrauterine growth restriction. Ultrasound obstet Gynecol 1997;9: 94-100.

[19] Parretti E, Mealli F, Magrini A, Cioni R, Mecacci F, Torre PL et al., Crosssectional and longitudinal evaluation of uterine artery Doppler velocimetry for the prediction of pre-eclampsia innormotensive women with specific risk factors Ultrasound Obstet Gynecol 2003; 22: 160-165.

[20] Afrakhteh M, Moeini A, Taheri MS, Haghighatkhah HR, Fakhri M, Masoom N. Uterine Doppler velocimetry of the uterine arteries in the second and third trimesters for the prediction of gestational outcome. Rev ras GinecolObstet 2014;36(1):35-9.

[21] Melchiorre K, Wormald B, Leslie K, Bhide A, ThilaganathanB.Firsttrimester uterine artery Doppler indicesin term and preterm pre-eclampsia. Ultrasound Obstet Gynecol 2008; 32: 133-137.

[22] Nagar T, Sharma D, Choudhary M, Khoiwal S, Nagar RP, Pandita A. The role of Uterine and Umbilical Arterial Doppler in High risk Pregnancy: A Prospective Observational Study from India. Clinical Medicine Insights: Reproductive Health Clin Med Insights Reprod Health. 2015 Apr 15;9:1-5.

[23] Crispi F, Llubra E, Dominguez C, Martin P et al., predictive value of angiogenic factors and uterine artery Doppler for early versus late onset preeclampsia and intrauterine growth restriction obstet Gynecol 2008;31:303-309. 